\title{
Correction to: Phase I dose escalation study of BI 836826 (CD37 antibody) in patients with relapsed or refractory B cell non-Hodgkin lymphoma
}

\author{
Frank Kroschinsky ${ }^{1}$ • Jan Moritz Middeke ${ }^{1} \cdot$ Martin Janz $^{2} \cdot$ Georg Lenz $^{3} \cdot$ Mathias Witzens-Harig $^{4}$. \\ Reda Bouabdallah ${ }^{5}$. Paul La Rosée $e^{6,7}$. Andreas Viardot ${ }^{8}$ • Gilles Salles ${ }^{9}$. Seok Jin Kim ${ }^{10} \cdot$ Tae Min Kim $^{11,12}$. \\ Oliver Ottmann ${ }^{13}$. Joerg Chromik ${ }^{14}$. Anne-Marie Quinson ${ }^{15}$. Ute von Wangenheim ${ }^{16}$. Ute Burkard ${ }^{16}$. \\ Andreas Berk ${ }^{17}$. Norbert Schmitz ${ }^{3}$ iD
}

Published online: 23 June 2020

(C) Springer Science+Business Media, LLC, part of Springer Nature 2020

\section{Correction: Invest New Drugs https://doi.org/10.1007/s10637-020-00916-3}

The original version of this article unfortunately contained an error. In the Conflict of interest statement, Anne-Marie Quinson is described as declaring no conflict of interest. In fact, as her affiliation shows, she is an employee of Boehringer Ingelheim Pharmaceuticals Inc. The corrected conflict of interest statement is shown below.

Conflict of interest Frank Kroschinsky reports receiving reimbursement for costs of study treatments, documentation, congress fees and travel expenses from Boehringer Ingelheim. Jan Moritz Middeke declares that he has no conflict of interest. Martin Janz declares that he has no conflict of interest. Georg Lenz reports personal fees and/or research support from Amgen, AstraZeneca, BMS, Abbvie, Bayer, Janssen, Novartis, Gilead /Kite, Celgene, Roche, Takeda, Morphosys, ACERTA, AQUINOX, Agios, Verastem, NanoString, and Springer, outside the submitted work. Mathias Witzens-Harig declares that he has no conflict of interest. Reda
Bouabdallah declares that he has no conflict of interest. Paul La Rosée reports personal fees from Boehringer Ingelheim (Travel support). Andreas Viardot reports personal fees and non-financial support from Roche, personal fees and non-financial support from Kite/Gilead, nonfinancial support from Abbvie, personal fees from Amgen, outside the submitted work. Gilles Salles reports personal fees from Amgen, BMS, Abbvie, Janssen, Merck, Novartis, Gilead / Kite, Epizyme, Pfizer, Celgene, Roche, Takeda, Autolus, MOrphosys, ACERTA, and Servier, outside the submitted work. Seok Jin Kim declares that he has no conflict of interest. Tae Min Kim declares that he has no conflict of interest. Oliver Ottmann declares that he has no conflict of interest. Joerg Chromik declares that he has no conflict of interest. Anne-Marie Quinson is an employee of Boehringer Ingelheim Pharmaceuticals Inc. Ute vonWangenheim and Ute Burkard are employees of Boehringer Ingelheim. Andreas Berk is an employee of ClinTriCare $\mathrm{GmbH} \& \mathrm{Co}$. KG, contracted by Boehringer. Norbert Schmitz declares that he has no conflict of interest.

Publisher's note Springer Nature remains neutral with regard to jurisdictional claims in published maps and institutional affiliations.

The online version of the original article can be found at https://doi.org/ 10.1007/s10637-020-00916-3

Norbert Schmitz

Norbert.Schmitz@ukmuenster.de

Extended author information available on the last page of the article 


\section{Affiliations}

\section{Frank Kroschinsky ${ }^{1} \cdot$ Jan Moritz Middeke ${ }^{1} \cdot$ Martin Janz $^{2} \cdot$ Georg Lenz $^{3} \cdot$ Mathias Witzens-Harig $^{4} \cdot$ Reda Bouabdallah $^{5}$. Paul La Rosée ${ }^{6,7}$. Andreas Viardot ${ }^{8}$. Gilles Salles ${ }^{9}$. Seok Jin Kim ${ }^{10} \cdot$ Tae Min Kim $^{11,12}$. Oliver Ottmann ${ }^{13}$. Joerg Chromik ${ }^{14}$. Anne-Marie Quinson ${ }^{15}$. Ute von Wangenheim ${ }^{16}$. Ute Burkard ${ }^{16}$. Andreas Berk ${ }^{17}$. Norbert Schmitz ${ }^{3}$ (10)}

1 Medical Department I, University Hospital at the Technical University of Dresden, Fetscherstr. 74, 01307 Dresden, Germany

2 Experimental and Clinical Research Center, Max Delbrück Center for Molecular Medicine and Charité - Universitätsmedizin Berlin, Robert-Rössle-Straße 10, 13125 Berlin, Germany

3 Department of Hematology and Oncology, University Hospital Muenster, Albert-Schweitzer-Campus 1, 48149 Münster, Germany

4 Internal Medicine V: Hematology, Oncology and Rheumatology, University Hospital Heidelberg, Im Neuenheimer Feld 672, 69120 Heidelberg, Germany

5 Department ofHematology, Institute Paoli Calmettes, 232 Boulevard de Sainte-Marguerite, 13009 Marseille, France

6 Klinik für Innere Medizin II, Universitätsklinikum, Jena, Germany

7 Klinik für Innere Medizin II, Schwarzwald-Baar-Klinikum, Villingen-Schweningen, Germany

8 Department of Internal Medicine III, University Hospital of Ulm, Albert-Einstein-Allee 23, 89081 Ulm, Germany

9 Department of Hematology, University Hospital of South Lyon, 165 Chemin du Grand Revoyet, 69310 Pierre-Bénite, France
10 Division of Haematology-Oncology, Department of Medicine, Samsung Medical Center, Sungkyunkwan University School of Medicine, 81 Irwon-ro, Irwon-dong, Gangnam-gu, Seoul, South Korea

11 Department of Internal Medicine, Seoul National University Hospital, 101 Daehak-Ro Jongno-Gu, Seoul 03080, South Korea

12 Cancer Research Institute, Seoul National University College of Medicine, 101 Daehak-ro, Jongno-gu, Seoul, South Korea

13 Division of Cancer and Genetics, Department of Haematology, Cardiff University, Heath Park, Cardiff CF14 4XN, UK

14 Universitätsklinikum Frankfurt, Johann-Wolfgang-GoetheUniversität, Theodor-W.-Adorno-Platz 1, 60323 Frankfurt, Germany

15 Boehringer Ingelheim Pharmaceuticals Inc., 900 Ridgebury Road, Ridgefield, CT 06877, USA

16 Boehringer Ingelheim Pharma GmbH \& Co. KG, Birkendorfer Str. 65, 88397 Biberach an der Riß, Germany

17 ClinTriCare GmbH \& Co. KG, Untere Illereicher Str. 10, 89281, Altenstadt, Germany 